\title{
Prenatal exposure to carbon monoxide delays postnatal cardiac maturation
}

\author{
Laura Sartiani ${ }^{1, *}$, Francesca Stillitano $^{2, *}$, Cristina Luceri ${ }^{2}$, Silvia Suffredini ${ }^{1}$, Simona Toti $^{2}$, Carlotta De Filippo ${ }^{2}$, \\ Vincenzo Cuomo ${ }^{3}$, Maria Tattoli ${ }^{4}$, Piero Dolara ${ }^{2}$, Alessandro Mugelli ${ }^{1,2}$ and Elisabetta Cerbai ${ }^{1,2}$
}

Prenatal exposure to toxicants, such as maternal smoking, may impair cardiovascular autonomic maturation in infants. We recently showed that exposure of pregnant rats to a mild concentration of carbon monoxide (CO), a component of cigarette smoke, delays postnatal electrophysiological maturation of ventricular myocytes from newborns rats, likely predisposing to life-threatening arrhythmias. To get a comprehensive view of developmental molecular abnormalities induced, at cardiac level, by prenatal CO exposure, we used microarray analysis approach on the rat heart at 4, 7 and 20 days postnatal life. The relationship between molecular and functional alterations was investigated by assessing the ventricular expression of $\mathrm{f}$-current, an electrophysiological marker of immature cardiac phenotype. Rats were prenatally exposed to 0 (CTR) or 150 p.p.m. CO and mRNA obtained from ventricular samples. Differential analysis and biological pathway analysis of microarray data were performed by using Newton's approach and the GENMAPP/MAPPFinder, respectively. The real-time RT-PCR reactions were performed by TaqMan probe-based chemistry. Freshly isolated patch-clamped ventricular cardiomyocytes were used to measure $I_{\mathrm{f}}$. Genes and pathways controlling cell cycle and excitation-contraction coupling were significantly modified in CO-exposed rats. The higher effect was observed in cardiomyocytes harvested from 7-day-old rats, in which mRNA expression for crucial sarcomeric proteins (myosin and actin subunits, troponin I), transporters $\left(\mathrm{Ca}^{2+}\right.$ transporting ATPase) and enzymes (aldolase) were significantly downregulated. Accordingly, the molecular and functional expression of $f$-channels, which represents a marker of fetal ventricular phenotype, was transiently greater in CO-exposed rats $(+200 \%)$ than in control ones. In conclusion, our study provides new insights into the molecular and functional mechanisms underlying cardiac maturation and its impairment by prenatal exposure to toxic components of smoking, such as CO.

Laboratory Investigation (2010) 90, 1582-1593; doi:10.1038/labinvest.2010.122; published online 19 July 2010

KEYWORDS: carbon monoxide; cardiomyocyte maturation; electrophysiology; f-channels; microarray; prenatal exposure

Congenital cardiac defects represent a major cause of premature death and morbidity in life births. Prenatal exposure to environmental toxicants, by altering the balance of factors turning on and off gene transcription, may influence susceptibility to congenital cardiac diseases. Although comprehension of these phenomena remains very limited, it is likely that exogenous disturbance of the combinatorial and/ or hierarchical action of genes involved in cardiac cell cycle may affect cardiogenesis or subsequent cardiac maturation.

Maternal smoking during pregnancy exposes the fetus to several toxicants including carbon monoxide (CO). Fetal hemoglobin has a fourfold higher affinity for CO compared with the adult form, ${ }^{1}$ thus increasing fetal exposure to $\mathrm{CO}$ damage. Exposure of pregnant rats to 150 p.p.m. CO results in blood levels of carboxy-hemoglobin comparable to those found in human cigarette smokers. ${ }^{2}$ By using a rat model of prenatal $\mathrm{CO}$ exposure, ${ }^{3,4}$ we previously showed that COexposed neonatal rats show functional hallmarks of delayed cardiomyocyte maturation, such as persistent prolongation in action potential duration and delayed upregulation of transient outward current. ${ }^{2}$ We postulated that these electrophysiological abnormalities, by destabilizing cardiac

\footnotetext{
${ }^{1}$ Centro Interuniversitario di Medicina Molecolare e Biofisica Applicata (C.I.M.M.B.A.), University of Firenze, Firenze, Italy; ${ }^{2}$ Department of Preclinical and Clinical Pharmacology, University of Firenze, Firenze, Italy; ${ }^{3}$ University of Rome 'La Sapienza', Rome, Italy and ${ }^{4}$ Department of Pharmacology and Human Physiology, University of Bari, Bari, Italy

Correspondence: Dr E Cerbai, PhD, Centro Interuniversitario di Medicina Molecolare e Biofisica Applicata (C.I.M.M.B.A.), Università degli Studi di Firenze, Viale G. Pieraccini 6, Firenze 50139, Italy

E-mail: elisabetta.cerbai@unifi.it

*These two authors contributed equally to this work.

Received 27 July 2009; revised 4 May 2010; accepted 12 May 2010
} 
repolarization, may create a transient susceptibility to arrhythmias. Indeed, maternal smoking during pregnancy is the major independent risk factor for sudden infant death. ${ }^{5}$ However, the molecular basis of this phenomenon has not been yet elucidated.

Perinatal cardiomyocyte growth is a developmentally crucial period, in which proliferation ceases and myocytes undergo binucleation, before terminal withdrawal from the cell cycle, and finally hypertrophy and maturation. ${ }^{6}$ Because of the complexity of the molecular events controlling postnatal cardiac maturation, we used a microarray analysis approach to get a comprehensive view of developmental changes induced by prenatal $\mathrm{CO}$ exposure on the heart at 4,7 and 20 days postnatal life. Moreover, the relationship between molecular and functional alterations was investigated by assessing the ventricular expression of f-current, as a marker of immature cardiac phenotype. Our results show for the first time that chronic fetal hypoxia caused by $\mathrm{CO}$ exposure impairs a number of critical pathways involved in postnatal cardiac development and checkpoints of cardiac cell cycle, leading to transient delay in maturation of molecular and functional properties.

\section{MATERIALS AND METHODS}

\section{CO Treatment of the Animals}

All the experiments were performed in accordance with the European Communities Council Directive of 24 November 1986 (86/609/EEC), for experimental animal care. Pregnant primiparous Wistar female rats (Harlan, Udine, Italy) were exposed to 0 or 150 p.p.m. CO mixed with air throughout the time of pregnancy as described earlier. ${ }^{3,4}$ This CO concentration is reported to result in carboxy-hemoglobin level similar to those attained in a moderate-heavy tobacco smoker. ${ }^{1}$

The duration of gestation was calculated starting from the day on which sperm was present in vaginal smears, taken on the morning at 09:00 hours after placement of two female with a single male rat. The day was designated as day 0 of gestation and for all rats the duration was 20-21 days. The number of living pups was similar in both groups. Within $24 \mathrm{~h}$ of birth, every litter was reduced to six male pups, and when necessary, female pups were used to reach the number of six pups. From 1 to 60 days of life, one control rat and one CO-exposed male rat were killed at any experimental time to maintain litter homogeneity. During weaning, male rats were kept apart from females, gathered together into two groups (control, Ctr and CO), which then were reduced to an equal number. All the rats survived until they were killed at the moment of the experiment.

\section{mRNA Extraction}

Total mRNA was isolated as described earlier. ${ }^{7,8}$ Briefly, tissue specimens were homogenized in OMNIzol reagent (Celbio). Genomic DNA was eliminated by incubating in DNase I. RNA was quantified by spectrophotometry. All $\mathrm{A}_{260} / \mathrm{A}_{280} \mathrm{~nm}$ ratios were above 1.8. Integrity was evaluated by ethidium bromide staining on a denaturing agarose gel. RNA samples were stored at $-80^{\circ} \mathrm{C}$ in RNAsecure resuspension solution (Ambion).

\section{Microarray}

For microarray analysis, we created a control and a COexposed pool of RNA for each experimental group (4, 7 and 20 days old rats) by mixing equal amount of RNA from each individual RNA sample; each pool was composed by four individual RNA samples.

Rat microarrays were constructed using the 'Rat Genome Oligo Set version 1.1' (Operon Technologies, CA, USA), composed of $70 \mathrm{mer}$ probes representing 5677 Rattus norvegicus genes, spotted with an OmniGrid 100 microarrayer (Genomic Solutions, Ann Arbor, MI, USA) into poly-L-lysine glass slides (Erie Scientific Company Portsmouth, NH, USA). After printing, the arrays were rehydrated with $0.5 \times$ SSC for $5 \mathrm{~min}$ and cross-linked with $60 \mathrm{~mJ}$ of energy using the BIO-LINK254 cross-linker (Discovery Scientific). For blocking, the slides were plunged in a solution containing $5.5 \mathrm{~g}$ of succinic anhydride dissolved in $335 \mathrm{ml}$ of 1-methyl-2-pyrrolidinone and $15 \mathrm{ml}$ of $1 \mathrm{M}$ sodium borate $\mathrm{pH} \mathrm{8.0,} \mathrm{for} 15 \mathrm{~min}$, under gently shaking. Then the slides were rinsed in boiled water, plunged in $95 \% \mathrm{EtOH}$ for $60 \mathrm{~s}$ and dried by spining $1 \mathrm{~min}$ at 550 r.p.m.

We performed three comparisons in duplicate: each pool of RNA from CO-exposed rat (labeled with Cy5) was compared with the control pool (labeled with Cy3; CyDye MonoReactive Dye Pack, Amersham, Cologno Monzese, Milan, Italy), using the indirect labeling method described by DeRisi (http://derisilab.ucsf.edu). Briefly, the reactive amine derivative of dUTP, 5-(3-aminoallyl)-2'-deoxyuridine 5-triphosphate (Sigma, Milan, Italy) was incorporated by Superscript II reverse transcriptase (Invitrogen, San Giuliano Milanese, Italy) and $20 \mu \mathrm{g}$ of total RNA using oligo dT (Invitrogen) and random examers (Roche, Monza, Milan, Italy). The ratio between aadUTP and dTTP was 2 aa-dUTP molecules to 3 dTTP molecules. After synthesis of cDNA, RNA was hydrolyzed by addition of sodium hydroxide and EDTA to a final concentration of 100 and $10 \mathrm{mM}$, respectively, and incubated at $65^{\circ} \mathrm{C}$ for $10 \mathrm{~min}$. The hydrolysis reaction was neutralized with $1 \mathrm{M}$ HEPES. After removing free nucleotides by purification, the aminoallyl-labeled samples were coupled to succinimidyl ester of Cy3 and Cy5 (Amersham, Milan, Italy). The hybridizations were performed at $63^{\circ} \mathrm{C}$ for $14-18 \mathrm{~h}$.

The images were scanned using a Genepix 4000B microarray scanner (Axon Instruments, Foster City, CA, USA); the loading of the array list (to locate the reporters on the array) and the image analysis, were performed with the GenePixPro6.1 software.

The following features were set to control spot quality: 60 pixel as minimum spot diameter; $50 \%$ as the minimum percentage of pixels for which the foreground intensity was greater than the background intensity +2 s.d.; 80 pixels as 
the minimum number of pixels; $20 \%$ as the maximum percentage of saturated pixels; 0.9 value for the ratio between the minimum and the maximum of the mean and the median of foreground intensity. The percentage of good spots in each array was $>65 \%$.

After background adjustment, for each gene we calculated the difference between the median foreground intensity and the background intensity, for each channel and the log-ratio of the background-adjusted intensity on the red channel vs green channel. Data were normalized by lowess function, calculated for each print-tip block, rescaling the log-ratios to have the same median-absolute-value (using the limma library of Bioconductor, http://www.bioconductor.org ${ }^{9}$ the information for each gene was expressed by averaging the log-ratio intensity of the two replicates).

Differential analysis was performed by Newton's approach $^{10}$ on normalized data, identifying genes significantly upregulated or downregulated $(P<0.001)$ with respect to the reference.

The Eisen software (freely available at http://rana.stanford. edu) was used to perform a cluster analysis using the $K$-means method. The analysis was performed on all genes, which passed the quality control step and were present in $100 \%$ of the experiments (3164 genes). Log-transformed fold changes were arbitrarily clustered into groups of genes having similar expression profiles. The number of nodes was chosen to give the largest possible number of similar or different patterns.

Biological pathway analysis was performed using the GenMAPP/MAPPFinder software tandem (http://www. genmapp.org). For GenMAPP, the 'Rn_Std_20070817' gene database and the 'Rn_20080619' pathway set were used. MappFinder analyses for pathways enrichment were performed for each group and for upregulated and downregulated genes, separately using a cutoff value for detecting a changed gene set at 1.4 or -1.4 to point out also minor but coordinated changes. The program calculates the $z$-score, a standardized difference score, determining the expected value and the s.d. of the number of genes meeting the criterion on an MAPP, taking into account the MAPP sizes. ${ }^{11}$ Only pathways showing a permute $P$-value $<0.05$ and a positive (enrichment) $z$-score were selected.

\section{Quantitative RT-PCR}

Reverse transcription reaction was performed by incubating a reaction mixture containing: $1 \mu \mathrm{g}$ RNA, $2.5 \mu \mathrm{M}$ random examers, $5.5 \mathrm{mM} \mathrm{MgCl}_{2}, 500 \mu \mathrm{M}$ each dNTPs, $0.4 \mathrm{U} / \mu \mathrm{l}$ RNase inhibitor, $1.25 \mathrm{U} / \mu$ l Multiscribe RT enzyme in a total of $100 \mu \mathrm{l} 1 \times$ reaction buffer. RT was performed at $25^{\circ} \mathrm{C}$ for $10 \mathrm{~min}, 48^{\circ} \mathrm{C}$ for $30 \mathrm{~min}$ and stopped by incubating at $95^{\circ} \mathrm{C}$ for $5 \mathrm{~min}$. All products were purchased from Applied Biosystem. The real-time RT-PCR reactions were performed using TaqMan Universal PCR Master Mix (Applied BioSystems) in a $20 \mu \mathrm{l}$ reaction volume containing $50 \mathrm{ng}$ of cDNA. All reactions were performed in triplicate and included a
Table 1 Primers and probes used for quantitative RT-PCR

\begin{tabular}{|c|c|c|}
\hline Gene & $\begin{array}{l}\text { Primers and } \\
\text { probes }\end{array}$ & Sequences \\
\hline & Foward & 5'-TGCTCACCAAGGGCAACAA-3' \\
\hline \multirow[t]{3}{*}{ HCN2 } & Probe & 5'-TGAAGCTGTCAGATGGCTCCTATITTG-3' \\
\hline & Reverse & 5'-CCTCGTGAGCAGGCAGATCT-3' \\
\hline & Foward & 5'-GAGCAGGAGAGGGTTAAGTCAGC-3' \\
\hline \multirow[t]{3}{*}{ HCN4 } & Probe & 5'-CCACCCCTACAGCGACTTCAGAПITACTGG-3' \\
\hline & Reverse & 5'-CAGCAACAGCATCGTCAGGT-3' \\
\hline & Foward & 5'-CTTCACCACCATGGAGAAGGC-3' \\
\hline \multirow[t]{2}{*}{ GAPDH } & Probe & 5'-CCTGGCCAGGTCATCCATGACAACTा--3' \\
\hline & Reverse & 5'-GGCATGGACTGTGGTCATGAG-3' \\
\hline
\end{tabular}

negative control. PCR reactions were performed using an ABI Prism 7500 Sequence Detection System (Applied Biosystems). Cycling conditions were: $2 \mathrm{~min}$ at $50^{\circ} \mathrm{C}, 10 \mathrm{~min}$ at $95^{\circ} \mathrm{C}$, and 40 cycles of $15 \mathrm{~s}$ at $95^{\circ} \mathrm{C}$ and $1 \mathrm{~min}$ at $60^{\circ} \mathrm{C}$. Relative quantification of mRNA levels was obtained by the 7500 system software, which uses the comparative method $(\triangle \mathrm{CT})$. Primers and TaqMan probes specific for HCN2, HCN4 and GAPDH were designed using PRIMER EXPRESS software (PE Applied Biosytems) (see Table 1).

Primers and probe for MiRP1 were obtained from ABI (Foster City, CA, USA) TaqMan Gene Expression Assay catalogue. This assay comes in a $20 \times$ reaction mix, spans an exon-exon junction, and is optimized to give $\sim 100 \%$ efficiency.

\section{Patch-Clamp Recordings}

Single left ventricular myocytes were isolated from Ctr or $\mathrm{CO}$-exposed rats according to a protocol based on previously described procedures ${ }^{2}$ and used within the day.

The experimental setup for patch-clamp (whole-cell) recording and data acquisition was similar to that described earlier. ${ }^{2}$ Experiments were performed simultaneously in two patch-clamp setups. The patch-clamped cell was superfused by means of a temperature-controlled microsuperfusor $\left(36-37^{\circ} \mathrm{C}\right)$, which allowed rapid changes of the solution bathing the cell, with normal or modified Tyrode's solutions. Patch-clamp pipettes had a resistance of 1.5-2.5 MOhm. After clamp steps to hyperpolarized membrane potential (usually $-120 \mathrm{mV}$ ), an $I_{\mathrm{f}}$ like current was recorded in most of the tested cells. $I_{\mathrm{f}}$ was considered to be present in a given cell when hyperpolarization elicited a time-dependent increasing inward current, which could be blocked by adding $4 \mathrm{mM}$ $\mathrm{CsCl}$. For the activation curve, $I_{\mathrm{f}}$ was evoked by hyperpolarizing steps (range: -50 to $-140 \mathrm{mV}$ ) from a holding potential of $-40 \mathrm{mV}$. 
Offline data analysis was performed with pClamp (version 9.0, Molecular Devices) and Origin 7.5 (MicroCal Software). All data are expressed as mean \pm s.e.m. Statistical analysis was performed by means of the Graph Pad Instat program, using the Student's $t$-test for grouped data or analysis of variance followed by Tukey's test for all other values. A probability value of $P<0.05$ was considered significant.

Concentrations are expressed in $\mathrm{mmol} / \mathrm{l}$. Normal Tyrode's solution (NT) contained $\mathrm{NaCl} 140, \mathrm{KCl} 5.4, \mathrm{CaCl}_{2} 1.5, \mathrm{MgCl}_{2}$ 1.2, glucose 5.5 and HEPES/NaOH 5 ( $\mathrm{pH} 7.35)$. Modified Tyrode's solution contained 4-aminopyridine $0.5, \mathrm{BaCl}_{2} 2$, $\mathrm{MnCl}_{2}$ 2, to block overlapping currents and $\mathrm{KCl} 25$ to amplify $I_{\mathrm{f}}$. Pipette solutions contained: K-aspartate $130, \mathrm{Na}_{2}$-ATP 5, $\mathrm{MgCl}_{2}$ 2, $\mathrm{CaCl}_{2}$ 5, EGTA 11 and HEPES-KOH 10 (pH 7.2; pCa 7.0).

\section{RESULTS}

\section{CO Treatment Is Associated with Changes in Several Pathways: Differential Analysis}

Our first attempt was to identify genes significantly upregulated and downregulated $(P<0.001)$ in each experimental group, by Newton's analysis (Figure 1a). The higher effect was observed in cardiomyocytes harvested from 7-day-old rats where we found the downregulation of myosin and actin subunits, troponin I and Atp2a2 (ATPase, $\mathrm{Ca}^{2+}$ transporting), which might predispose to myofilament dysfunction, although no functional data are available at present. A significant downregulation was also observed for the expression of IGF-2, a growth factor involved in heart development, ${ }^{12}$ for aldolase, that controls the first critical step of cardiomyocyte glycolisis and for the ribosomal S6 gene, whose phosphorylation is one of the early intracellular responses to growth factor stimulation in both invertebrates and vertebrates.

In cardiomyocytes harvested from 4-day-old rats, there were only few genes differentially expressed compared with unexposed rats; among them we found another gene codifying for an ATPase subunit, the Atpla3 (ATPase, $\mathrm{Na}^{+} / \mathrm{K}^{+}$ transporting). In 20-day-old rats $\mathrm{CO}$ exposed, we did not find genes differentially expressed compared with controls. The results of this analysis are showed in Table 2 .

\section{Cluster Analysis}

$K$-mean clustering of the microarray data showed a large number of genes overexpressed in all CO-exposed animals, compared with rats of the same age (908 genes); a cluster of 535 genes was upregulated in 4-day-old rats (cluster 1; Figure 1b) and a cluster of 728 genes was downregulated in 7-day-old rats (cluster 2; Figure 1b).

In the cluster of genes overexpressed in CO-exposed 4-dayold rats, we found several cytochromes (Сyp2a3a, Cyp2b21, Cyp2c23, Cyp2d3 and Cyp4b1), gluthatione $S$-transferases (Gstm1, Gstm3 and Gstt2), potassium and calcium channel subunits (Kcna1, Kcna3, Kcnb2, Kcne3, Kcnh8, Kcnj1, Kcnj2, Kcnj3, Kcnk3, Cacna2d1, Cacnb1 and Cacng5) and phosphodiesterases (Pde10a, Pde3b, Pde8a and Pde9a). a
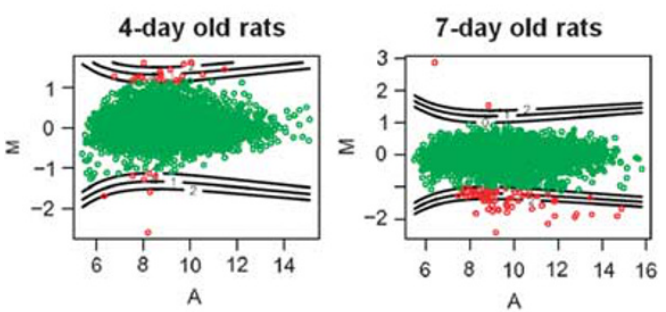

20-day old rats

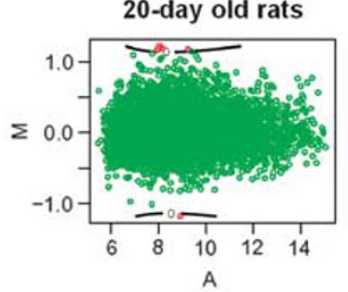

b

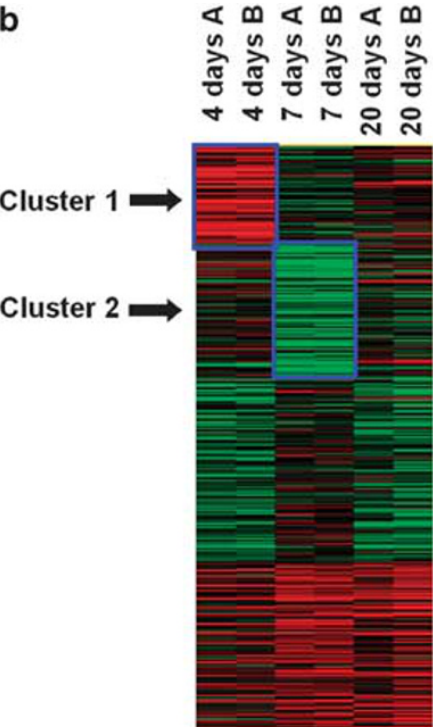

Figure 1 (a) Distribution of spot fluorescence intensities in microarray hybridization of mRNA extracted from cardiomyocytes of rats prenatally exposed to $\mathrm{CO}$ in comparison with cardiomyocytes from unexposed rats. $R$ represents red fluorescence (Cy5) and $\mathrm{G}$ represents green fluorescence (Cy3); $x$ axis: values of $A=\left(\log \left(R^{\star} G\right)\right.$ and $y$ axis: values of $M=(\log (R / G)) 0.5$. In each plot, red spots outside the last line represent upregulated or downregulated genes $(P<0.01) ;$ green spots and red spots inside the lines represent genes with no significant expression variations. (b) $K$-mean cluster of the microarray data. The analysis was performed on all genes, which passed the quality control and were present in all the experiments (3164 genes); $\mathbf{a}$ and $\mathbf{b}$ are technical replicates of the same comparison. 
Table 2 Genes differentially expressed by Newton's analysis $(P<\mathbf{0 . 0 0 1})$

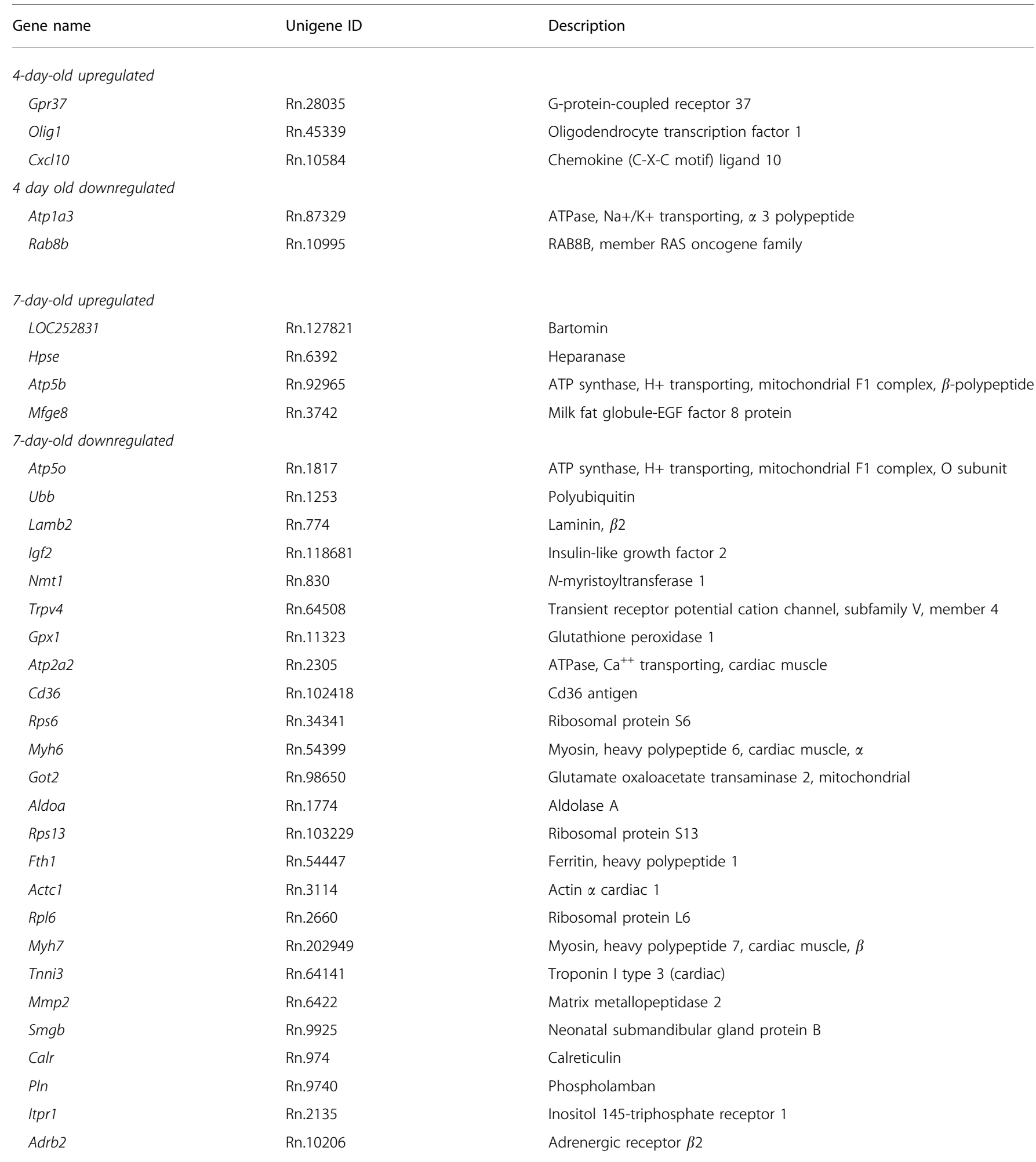

In the cluster of genes downregulated in CO-exposed 7-day-old rats, we noted several genes belonging to the actin and myosin protein family (Acta1, Acta2, Actb, Actg2 and Actn4, and Myh2, Myh13, Myh6, Myh7, Myh9, Myl3, Mylk2,
Myole and Myo5a), annexins (Anxa1, Anxa2 and Anxa5), ATP synthases (Atp5a1, Atp5b, Atp5f1, Atp5g2, Atp5i, Atp5j and Atp5o), calcium channel subunits (Cacnb2, Cacng5, Cacng6, Camk2a, Camk2n2 and Camkk1), procollagen 
subtypes (Col1a1, Col1a2, Col2a1, Col3a1 and Col5a2), members of the respiratory chain (Cox4i1, Cox6a2, Cox7a2, Cox8a and Cox8h), integrins (Itgam, Itgb1 and Itgb3), members of the ubiquitin/proteasome system (Psmb2, Psmb4, Psmb6, Psmc2, Psmc4, Psmd1, Psme1, Ubb, Ubelc and Ube2i), a large number of ribosomal proteins (Rpl10a, Rpl13, Rpl14，Rpl15，Rpl21，Rpl27，Rpl28，Rpl29，Rpl31，Rpl35a, Rpl36, Rpl36a, Rpl37, Rpl39, Rpl4, Rpl41, Rpl6, Rpn1, Rps11, Rps13, Rps14, Rps15, Rps15a, Rps17, Rps2 Rps24, Rps26, Rps29 and Rps6) and troponins (Tnnil, Tnni3 and Tnnt2).

\section{Pathway Analysis}

To gain further insight to microarray results, we performed pathways analysis. In the cardiomyocytes of rats prenatally exposed to CO, GenMAPP and MAPPFinder analysis identified several pathways upregulated or downregulated, compared with the control group (Figure 2). As expected, the results of this analysis were in line with those obtained with cluster analysis; moreover, the analysis identified several differential expression patterns suggestive of modifications in relevant biological pathways.

More specifically, in 4-day-old rats, functional analysis showed a significant downregulation of genes associated with the apoptotic process, cell cycle and RNA transcription such as CASP 9, CASP2, Bcl-2-like 1, cyclin-dependent kinase inhibitor 1, histone deacetylase 3, transcription factor E2F5, M-phase inducer phosphatase 2 and Cdkn1A (also known as p21), an inhibitor of cell proliferation whose upregulation

a
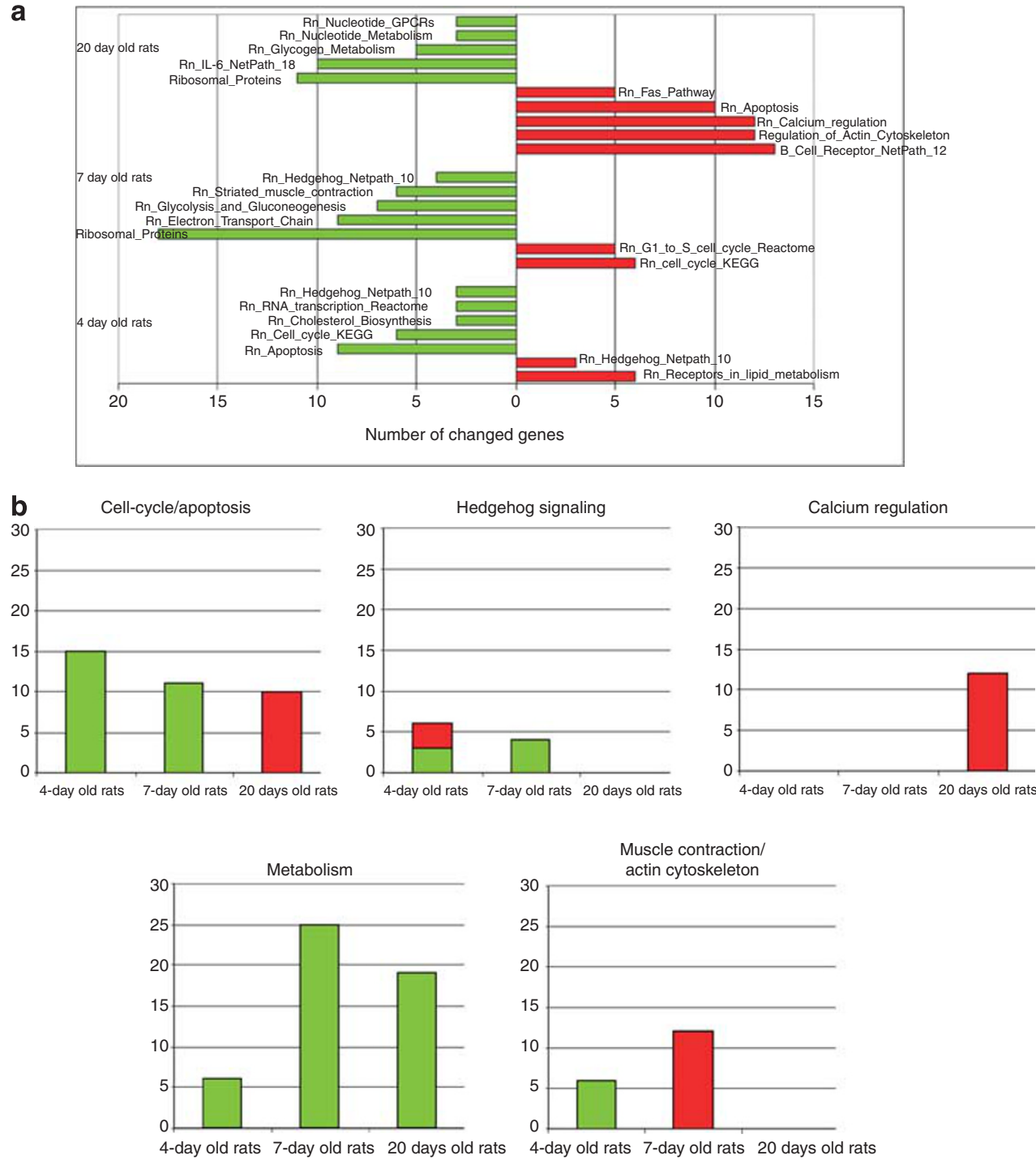

Figure 2 Pathways significantly modulated in cardiomyocytes isolated from rats exposed prenatally to CO, compared with non-exposed controls. (a) Results of the pathway analysis performed using GenMAPP/MAPPFinder software. Pathways significantly enriched ( $z$ score $>1.97)$ in downregulated genes are represented on the left (in green) and pathways significantly enriched in upregulated genes are on the right (in red). (b) Number of genes upregulated (in red) or downregulated (in green) associated to selected biological processes, found to be modulated in CO-exposed rats compared with unexposed animals. 
after birth promotes cell cycle exit. Significant alterations were also found in the metabolism map, with several nuclear receptors and cytochromes upregulated (Cyp7a1, Cyp4b1, Cyp8b1 and Cyp2c7), and the Hedgehog (Hh) signaling map.

In 7-day-old rats, besides the Hh signaling map, several genes associated with ribosomal proteins, electron transport chain, glycolysis and gluconeogenesis and sarcomeric proteins (troponin I, troponin T, myosin-6, $\alpha$-actinin-4, actin, $\alpha$-cardiac muscle 1) were downregulated.

In 20-day-old rats, the only significantly downregulated pathways were found in the glycogen metabolism and the IL6 signaling map, whereas the Fas pathway, the cytoskeleton and the cardiac cell calcium map were upregulated (Figure 2).

\section{Sustained Functional Expression of $\mathbf{f}$-Channels in CO-exposed Neonatal Rats}

Microarray analysis suggests that rat offspring of mothers exposed to $\mathrm{CO}$ shows abnormalities in several expression pathways. On the basis of previous electrophysiological evidence, ${ }^{2}$ we hypothesized that these alterations hint at a delayed postnatal maturation. To get a functional correlate, we evaluated the temporal pattern of expression of f-current. In fact, $I_{\mathrm{f}}$ is overexpressed in rodent ventricular myocytes during the fetal life but is downregulated after birth ${ }^{13,14}$; eventually, $I_{\mathrm{f}}$ expression is reinduced when adult cardiomyocytes undergo 'pathological' hypertrophic growth such as in cardiac diseases. ${ }^{8}$ Figure 3 shows typical action potentials (upper panels) and currents (lower panels) recorded in myocytes isolated from control and CO-exposed rat hearts at 2 (panels a and b) and 40 (panels $\mathrm{c}$ and d) days after birth. It is evident that the immature action potentials exhibit a longer duration and a prominent diastolic depolarization, giving rise to spontaneous activity (Figure 3a, arrows); this feature is associated with the presence of a large time-dependent f-current (Figure 3b). With postnatal maturation, action potential shortens more strikingly in control than in CO-exposed rats (as reported earlier ${ }^{2}$ ); simultaneously, f-current becomes almost undetectable in control but it remains markedly larger in cells from COexposed rats.

Figure 4a summarizes data on $I_{\mathrm{f}}$ density, measured at $-120 \mathrm{mV}$ : at $2-8$ days after birth (early), maximal current density was similar in control and CO-exposed rats $(2.4 \pm 0.4 \mathrm{pA} / \mathrm{pF}, n=39 \quad$ vs $2.7 \pm 0.4 \mathrm{pA} / \mathrm{pF}, n=29$; n.s.). However, in older CO-exposed rats (middle, 10-20 days), it persisted to values similar to those measured in perinatal myocytes $(2.4 \pm 0.3 \mathrm{pA} / \mathrm{pF} ; n=17)$ whereas, in control rats, current density rapidly decreased to values typical of adult cardiomyocytes (eg, $50-60$ days, late) $(0.8 \pm 0.4 \mathrm{pA} / \mathrm{pF}$, $n=23)$. I $I_{\mathrm{f}}$ activation kinetics was measured by monoexponential fitting of current traces evoked at $-120 \mathrm{mV}$ (Figure 4b) in control and CO-exposed rats; no statistically significant differences were observed at any age, although a tendency toward slower activation was observed at 20-30 days, that is in correspondence of a relatively higher $I_{\mathrm{f}}$ density in CO-treated $v s$ control rats. Typical recordings of $I_{\mathrm{f}}$ elicited by steps to increasing negative potentials $(-60$ to $-140 \mathrm{mV}$ ) obtained in myocytes from 4-week-old control and COtreated rats are reported in Figure $4 \mathrm{c}$ and d, respectively.

Myocytes isolated from CO-exposed pups were not altered in size. Indeed, the average values of membrane capacitance,
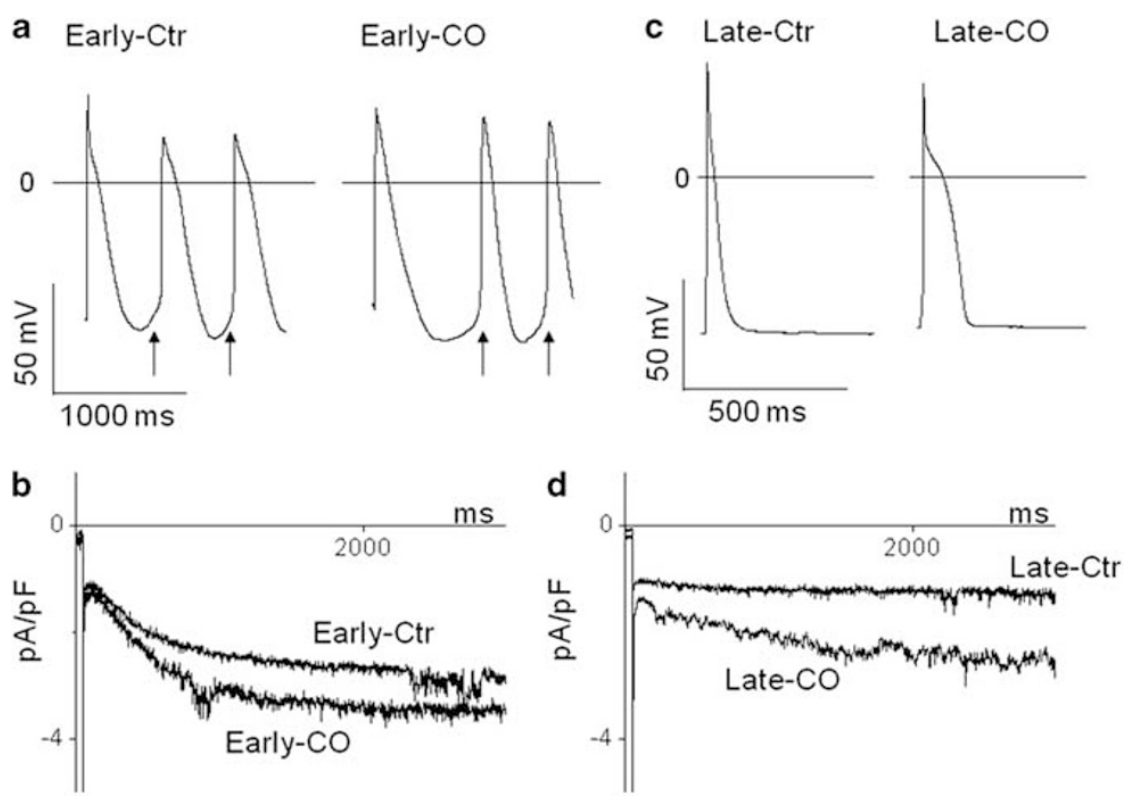

Figure 3 Postnatal electrophysiological maturation of rat cardiomyocytes. Action potentials (upper panels) and f-currents (lower panels) recorded in myocytes isolated from control and CO-exposed rat hearts at 2 (panels a, b) and 40 (panels c, d) days after birth. 
a
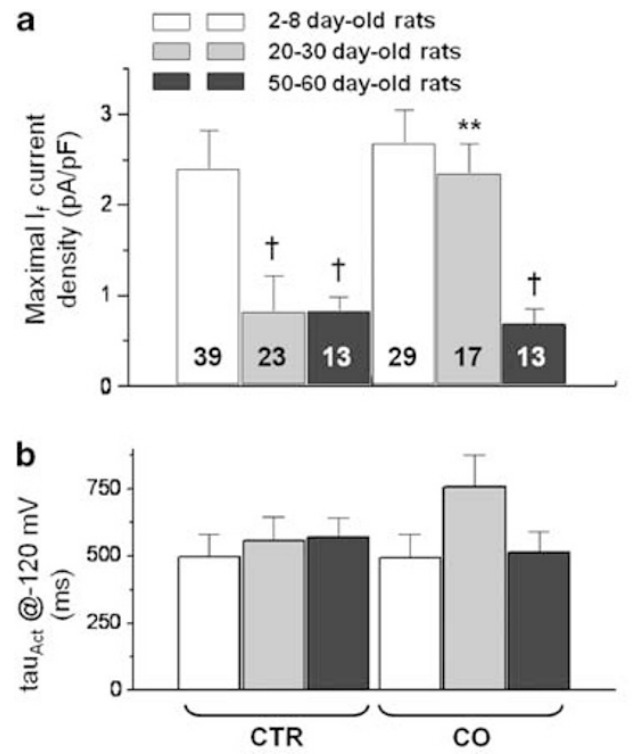

C

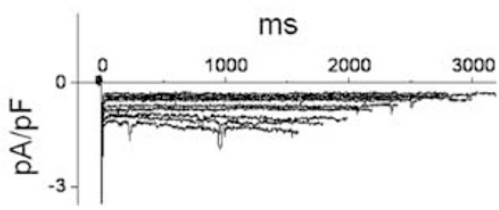

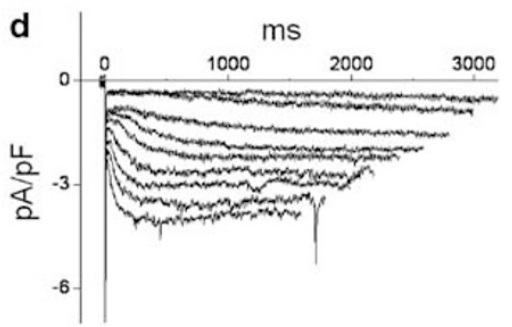

Figure $4 I_{\mathrm{f}}$ density (a) and activation constant (b), measured at $-120 \mathrm{mV}$, in cardiomyocytes at different stages of postnatal maturation, in CO-exposed (CO) and non-exposed control rats (CTR), ${ }^{\dagger} P<0.05$ vs 2 -8-day-old rats; ${ }^{* *} P<0.01$ vs age-matched $C$ tr. (c, d) Typical recordings of $I_{\mathrm{f}}$ elicited by steps to increasing negative potentials ( -60 to $-140 \mathrm{mV}$ ) obtained in myocytes from 20-day-old control and CO-treated rats, respectively.
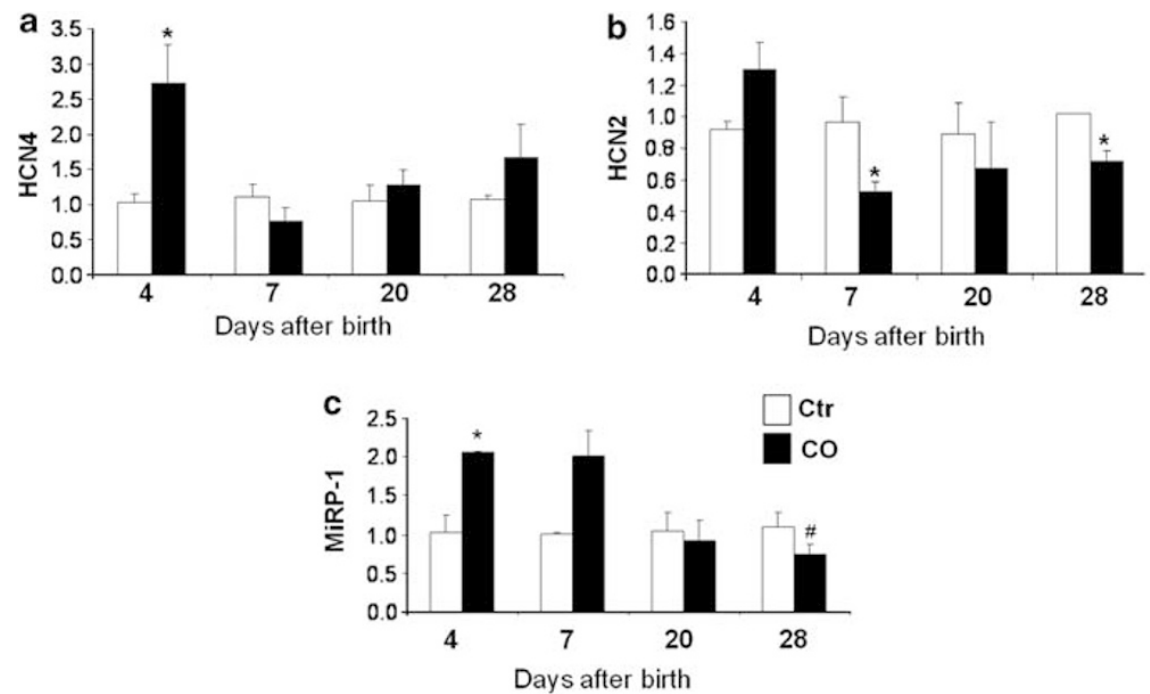

Figure 5 Relative expression of HCN4 (a) HCN2 (b) and MiRP-1 (c) mRNA in control (Ctr) and CO-treated samples by TaqMan RT-PCR. Quantification was performed at 4, 7, 20 and 28 days after birth. Each column represents the mean of 3 different samples in triplicate \pm s.e.m. ${ }^{\star} P<0.05$ vs Ctrl; ${ }^{\#} P<0.01$, linear trend.

an electrophysiological index of cell size, measured in myocytes from CO-treated rats was not statistically different from age-matched controls (early: $15.2 \pm 0.9 \mathrm{pF}, n=37$ vs $16.6 \pm$ $1.6 \mathrm{pF}, n=38$, n.s.; middle: $57.9 \pm 4.1 \mathrm{pF}, n=26$ vs $58.2 \pm 4.7 \mathrm{pF}$, $n=27$, n.s.; late $134.8 \pm 11.1 \mathrm{pF}, n=25$ vs $122.1 \pm 13.6 \mathrm{pF}$, $n=22$, n.s.).

\section{Molecular Properties of $\mathrm{f}$-Channels in CO-Exposed Neonatal Rats}

The $\alpha$-subunit of the f-channel is coded by the hyperpolarization-activated cyclic nucleotide gated $(\mathrm{HCN})$ gene; among the four isoforms, HCN4 is typical of fetal ventricular myocytes but is rapidly downregulated during cardiac maturation..$^{7,14,15}$ A higher expression of $\mathrm{HCN} 4$ was detected in CO-exposed rat hearts at 4 days after birth $(2.73 \pm 0.54 \mathrm{vs}$ $1.04 \pm 0.11$ ) (Figure 5a); this was accompanied by a relative upregulation of the mRNA coding for MinK-related peptide 1 (MiRP-1) $(2.06 \pm 0.02$ vs $1.03 \pm 0.2$ at 4 days) (Figure $5 \mathrm{c}$ ). MiRP-1 is a $\beta$-subunit that enhances the expression and speeds up the kinetics of activation of the $\mathrm{HCN}$-derived proteins; in the adult heart, MiRP-1 is expressed in the sinus node region but hardly detectable in the ventricle. ${ }^{16} \mathrm{HCN} 2$, whose density has been reported to increase in postnatal ventricular maturation with a peak between 10 and 20 days of 
age, ${ }^{17}$ was significantly less expressed in CO-treated $v s$ Ctr hearts at $7(0.53 \pm 0.06 v s 1.08 \pm 0.2)$ and $28(0.72 \pm 0.07 v s$ $1.02 \pm 0.001$ ) days (Figure $5 b$ ).

\section{DISCUSSION}

In a rat model of prenatal exposure to $\mathrm{CO}$, we showed for the first time that this condition is associated with significant alterations in the expression of several pathways and genes controlling postnatal cardiac development. In agreement with our earlier findings, ${ }^{2}$ present results are suggestive of a delayed maturation of the neonatal heart. In particular, pathways controlling cell cycle and excitation-contraction coupling are significantly modified in $\mathrm{CO}$-exposed rats. Accordingly, the molecular and functional expression of f-channels, which represents a marker of fetal ventricular phenotype, is transiently greater in CO-exposed rats than in control ones.

\section{Molecular Hallmarks of Delayed Cardiac Cell Maturation Because of Prenatal CO Exposure}

Cardiomyocytes lose their ability to proliferate soon after birth although they undergo a final round of incomplete cell division associated with DNA synthesis. ${ }^{6}$ This phase peaks at days 4-6 after birth, and by 3 postnatal weeks $90 \%$ of rodent myocytes are binucleated. ${ }^{18}$ A similar temporal pattern has been estimated to occur in humans. ${ }^{19}$ Taken together, these data suggest that the first postnatal weeks represent a temporally crucial period where any perturbation of the coordinated molecular events controlling cardiomyocyte maturation may lead to harmful functional abnormalities.

Our microarray data show that in 4- and 7-day-old rats, a significant upregulation or downregulation of several key modulators of cell cycling occur as a consequence of prenatal exposure to CO (G1-to-S cell cycle control pathway and cell cycle pathway). Of note, Cdkn1A (also known as p21), an inhibitor of cell proliferation whose upregulation after birth promotes cell cycle exit, ${ }^{6}$ was downregulated in prenatally CO-exposed rats compared with controls. Interestingly, myocyte hyperplasia was reported to occur in a rat model exposed to similar CO concentration (200 p.p.m.), ${ }^{20}$ thus suggesting that withdrawal of postnatal cardiomyocytes from the cell cycle may be hampered or delayed by fetal $\mathrm{CO}$ exposure.

Among the downregulated pathways, we found the $\mathrm{Hh}$ signaling process (at 4 and 7 days postnatal life). The $\mathrm{Hh}$ proteins act as morphogens in many tissues during embryonic development. Among the three highly conserved mammalian $H h$ genes, Sonic hedgehog (Shh) is the most widely expressed during development ${ }^{21}$; the transmission of Shh signaling is accomplished through its receptor Patched1 (Ptc1) and Smoothened (Smo). Shh and Ptcl genes were both downregulated in the heart of 4- and 7-day-old rats exposed to CO prenatally compared with controls. These effects are of interest considering that the Shh deficiency in mice is embryonically lethal, leading to multiple defects beginning in early-to-mid gestation. ${ }^{22}$ Moreover, evidence suggests a role for the Shh signaling pathway in heart morphogenesis; Shh-1- display a heart-looping defect ${ }^{23,24}$ and Ptc1-1mice exhibit heart morphological defects. ${ }^{25}$ It has been also reported that Shh signaling through Gli1/2 is sufficient to initiate the expression of cardiac muscle transcription factors, leading to cardiomyogenesis in stem cells toward a cardiac muscle lineage ${ }^{26}$ thus supporting an involvement for Shh in regulating this process.

Other significant alterations found in $\mathrm{CO}$-exposed rats are suggestive of potential functional consequences on heart maturation. The ribosomal protein pathway was the most affected at 7 and 20 days after birth. Although the present knowledge does not allow to extrapolate a functional role for most of these proteins, it is worth noting that phosphorylation of $40 \mathrm{~S}$ ribosomal protein S6 is one of the early intracellular responses to growth factor stimulation and that impairment of this mechanism causes perinatal lethality in mice. ${ }^{27}$

Downregulation of metabolic pathways also deserves some attention. Soon after birth, enzymes involved in cardiac glucose/lactate oxidation and fatty acid oxidation (which provides the major source of fuel in the adult heart) undergo profound remodeling. In this respect, we observed abnormalities in two critical pathways. The glycolytic pathway was significantly downregulated at 7 days, and one of the most affected genes was aldolase $\mathrm{A}$, which has been reported to undergo physiological postnatal increase in ovine. ${ }^{28}$

In the same line, several genes in the electron transport chain were significantly altered by CO-exposure at 4 and 7 days. Member of this pathway were genes coding for subunits of the cytochrome C oxidase complex (COX5a) and ATP synthase complex (ATP5), which were significantly downregulated. As for aldolase, COX5a has been reported to increase postnatally in parallel with the switch from anaerobic to aerobic metabolism. ${ }^{28}$ Thus, alterations in metabolic pathways may be a further expression of delayed postnatal maturation. However, $\mathrm{CO}$ acts at several heme-proteins and cytochromes during acute exposure, ${ }^{29}$ therefore it is also possible that these changes reflect adaptation to chronic impairment of myocardial cytochrome oxidase activity. Overall, their functional correlation remains speculative as an evaluation of metabolic features of cardiomyocytes from $\mathrm{CO}$-exposed rats was not attempted at this stage.

\section{Delayed Functional Maturation Because of CO-Exposure}

In an earlier study performed in the same model, electrophysiological properties of patched-clamped left ventricular cardiomyocytes from $\mathrm{CO}$-exposed rat hearts were suggestive of a delayed maturation toward the adult phenotype. ${ }^{2}$ This hypothesis was based on the observation that action potential duration was persistently prolonged at 4 weeks after birth, but already shortened to values similar to the adult rat ventricular AP in control rats. This feature was paralleled by a delayed maturation of the transient outward current, that 
undergoes a marked postnatal upregulation. ${ }^{30,31}$ Present data seem to confirm this hypothesis: at 7 days after birth, Myh6 coding for $\alpha$-myosin heavy chain and Actcl coding for $\alpha$-actin, which replace, respectively, the $\beta$-myosin and the skeletal actin isoforms typical of the fetal rodent heart, ${ }^{32}$ are less expressed in CO exposed than in control hearts. Similarly, the cardiac isoforms of regulatory sarcomeric proteins troponin $\mathrm{T}$, troponin I and myosin light chain $(\mathrm{Myl})$, whose expression is positively modulated in the postnatal period, ${ }^{33}$ are less expressed in $\mathrm{CO}$ exposed than in control rat hearts whereas the skeletal isoform of troponin $\mathrm{T}$ and the smooth muscle isoform Myl9 are upregulated in CO-exposed neonatal rats.

Alike, alterations in the calcium regulatory pathway were detected: in particular, calreticulin and IP3 receptor are upregulated and phospholamban downregulated in CO-exposed rat hearts. Calreticulin expression is high in the developing heart, but postnatal downregulation of the gene in the heart occurs ${ }^{34}$ and elevated cardiac expression of the protein in transgenic mice after birth leads to arrhythmias and sudden death. ${ }^{35}$ Phospholamban mRNA increases eightfold from fetal to adult stages, in parallel with the increase of sarcoplasmic reticulum function. ${ }^{36}$

\section{$I_{\mathrm{f}}$ Expression in Postnatal Development}

Pacemaker activity, which is restricted to sinoatrial node in the adult life, is present in neonatal and embryonic ventricular cardiomyocytes. This phenomenon is paralleled by an elevated $I_{\mathrm{f}}$ current density that decreases during maturation in rats, the highest value being measured at 1-2 days and then gradually diminishing reaching a steady-state value at around 1 month of age. ${ }^{13}$ Something similar occurs in mice, but high $I_{\mathrm{f}}$ expression is detected only during fetal life, in parallel with high $\mathrm{HCN} 4$ expression, and tends to decrease already before birth. ${ }^{14}$ The latter observation is very consistent with more recent data on cardiac morphogenesis, showing that HCN4 is widely and highly expressed in the immature cardiac crescent in the mouse, and progressively restricted to the nodal areas thereafter being repressed in the ventricular and atrial chambers. ${ }^{37}$ No data exist for human neonatal ventricular cardiomyocytes; however, HCN4 expression decreases from early to late maturation stage in cardiomyocytes derived from human embryonic stem cells. ${ }^{7}$ Therefore, $I_{\mathrm{f}}$ occurrence can be considered as a hallmark of immaturity for ventricular cardiomyocytes. We have demonstrated earlier that the properties of cardiomyocytes isolated from CO-exposed rat during in utero life are consistent with a delayed electrophysiological maturation. ${ }^{2}$ Present results confirm and extend those data. In fact, $I_{\mathrm{f}}$ density is persistently higher in 4-week-old rats, and the mRNA level of HCN4 and MiRP1 (coding for the $\alpha$ - and $\beta$-subunit of the channel) are higher at birth in CO-exposed vs control rats.

In the rat ventricle, $I_{\mathrm{f}}$ can be recorded during the first days after birth, but its expression decreases rapidly thereafter and, at 4 weeks after birth, $I_{\mathrm{f}}$ density is similar to that measured in adult ventricular cells. ${ }^{13}$ A maturation-dependent decrease of HCN4 expression associated with a progressive reduction in the rate of spontaneous beating and steepness of diastolic depolarization has been also detected in human cardiomyocytes derived from embryonic stem cells. ${ }^{7}$ Taken together, these data suggest that the persistence of f-current in 4-weekold rat cardiomyocytes is consistent with a delayed acquisition of a mature phenotype. In contrast to current density, the observed modifications in $H C N$ and MiRP-1 expression levels in CO-treated vs control rats did not result in a significant alteration of current kinetics. Gating properties of native f-channels cannot be easily extrapolated from the relative proportion of $H C N$ isoforms and/or MiRP-1, as other factors may have a role (as discussed in the next section).

\section{Limitations of the Study and Clinical Implications}

Unfortunately, we could not perform ECG in the rats therefore we cannot exclude abnormal electrical behavior at the whole heart level. Nonetheless, there were no differences in mortality or weight gain between rats exposed to $\mathrm{CO}$ and controls. ${ }^{38}$ The explanation is not obvious, but it is important to underline that a latent 'arrhythmogenic propensity', such as reduction of repolarization reserve or destabilization of diastolic potential, often results in arrhythmias only in the presence of physiological triggering factors, such as adrenergic overactivation or stress conditions. ${ }^{39,40}$ Moreover, at variance with channelopathies because of mutations, the alterations that we observed in hearts from CO-exposed rats are not permanent, but rather spontaneously normalize toward control during cardiac maturation. To explore the occurrence and/or the susceptibility to develop arrhythmias, further investigation is warranted to assess the effect of stressors on ECG and/or mortality within the 'period of vulnerability'.

We did not observe significant differences in $I_{\mathrm{f}}$ density in early postnatal myocytes, that is at the stage when differences between $\mathrm{CO}$ and $\mathrm{Ctr}$ rats concerning mRNA levels of HCN4 and other genes detected by qPCR or microarray functional analysis, were maximal. However, it must be recalled that f-channels result from assembling of different isoforms, and the exact stoichiometry is unknown; although the expression of HCN1 and HCN3 in rat ventricular myocytes seems to be very low or undetectable, ${ }^{41}$ we cannot exclude the contribution of other isoforms to the persistent upregulation of $I_{\mathrm{f}}$. Furthermore, a posttranscriptional regulation of $\mathrm{HCN}$ by microRNA $(m i R)$ has been shown ${ }^{42}$ that may at least partially account for changes in $I_{\mathrm{f}}$ density during development. Modulation of postnatal expression of $m i R$ by $\mathrm{CO}$ and toxicants is an intriguing novel field that deserves further investigation. Finally, f-channel properties seem to be regulated by sublocalization in membrane caveolae and interaction with caveolin- $3 ;^{43}$ as caveolin expression represents a crucial step in cardiomyocyte maturation, ${ }^{44}$ we cannot 
exclude that altered or delayed channel trafficking may result in different current expression and function.

Because of species differences, any extrapolation to the human setting has to be cautiously drawn. Notwithstanding these limitations, our study may have relevant implications. In fact, beside cardiomyocytes, ${ }^{2}$ central and peripheral neurons may be target of CO-mediated damage as shown by impaired myelin deposition and SNC retardation in the same rat model. ${ }^{3}$

Fatal acute CO intoxication at low CO saturation levels $(<20 \%)$ has been reported for fetuses and infants ${ }^{45}$ as fetal hemoglobin has a greater affinity (up to eightfold) for CO than adult hemoglobin. ${ }^{46}$ This susceptibility decreases slowly after 6 months as adult hemoglobin synthesis is activated whereas fetal hemoglobin synthesis is deactivated. Maternal tobacco use (and possibly environmental smoking), as major source for $\mathrm{CO}$, has been linked to intrauterine growth retardation, prematurity and congenital malformations, including cardiac defects. ${ }^{47}$ However, even in the absence of structural heart disease, perinatal mortality is significantly higher in neonates from smoking mothers, thus suggesting that alterations elusive to autopsy may account for these deaths.

\section{ACKNOWLEDGEMENTS}

This work was supported by a grant from the Scottish Cot Death Trust (UK) and European Union (LSH M/CT/2006/018676). Electrophysiological and molecular biology equipment was kindly provided by a grant from Ente Cassa di Risparmio di Firenze (Italy).

\section{DISCLOSURE/CONFLICT OF INTEREST}

The authors declare no conflict of interest.

1. Aubard Y, Magne I. Carbon monoxide poisoning in pregnancy. BJOG 2000;107:833-838.

2. Sartiani L, Cerbai E, Lonardo G, et al. Prenatal exposure to carbon monoxide affects postnatal cellular electrophysiological maturation of the rat heart. A potential substrate for arrhythmogenesis in infancy. Circulation 2004;109:419-423.

3. Mereu G, Cammalleri M, Fa M, et al. Prenatal exposure to a low concentration of carbon monoxide disrupts hippocampal long-term potentiation in rat offspring. J Pharmacol Exp Ther 2000;294:728-734.

4. Carratù MR, Cagiano R, Desantis $\mathrm{S}$, et al. Prenatal exposure to low levels of carbon monoxide alters sciatic nerve myelination in rat offspring. Life Sci 2000;67:1759-1772.

5. Sullivan FM, Barlow SM. Review of risk factors for sudden infant death syndrome. Paediatr Perinat Epidemiol 2001;15:144-200.

6. Ahuja P, Sdek P, MacLellan WR. Cardiac myocyte cell cycle control in development, disease, and regeneration. Physiol Rev 2007:87:521-544.

7. Sartiani L, Bettiol E, Stillitano F, et al. Developmental changes in cardiomyocytes differentiated from human embryonic stem cells: a molecular and electrophysiological approach. Stem Cells 2007;25:1136-1144.

8. Stillitano F, Lonardo $G$, Zicha $S$, et al. Molecular basis of funny current (If) in normal and failing human heart. J Mol Cell Cardiol 2008;45:289-299.

9. Gentleman R, Huber W, Carey V. Bioinformatics and Computational Biology Solutions Using R and Bioconductor. Springer: London, 2005.

10. Newton MA, Kendziorski CM, Richmond CS. On differential variability of expression ratios: improving statistical inference about gene expression changes from microarray data. J Comput Biol 2001; 8:37-52.
11. Doniger S, Salomonis N, Dahlquist K. MAPPFinder: using Gene Ontology and GenMAPP to create a global gene-expression profile from microarray data. Genome Biol 2003;4:R7.

12. Morali OG, Jouneau A, McLaughlin KJ, et al. IGF-II promotes mesoderm formation. Develop Biol 2000;227:133-145.

13. Cerbai E, Pino R, Sartiani $L$, et al. Influence of postnatal-development on if occurrence and properties in neonatal rat ventricular myocytes. Cardiovasc Res 1999;42:416-423.

14. Yasui K, Liu W, Opthof $\mathrm{T}$, et al. I(f) current and spontaneous activity in mouse embryonic ventricular myocytes. Circ Res 2001;88: 536-542.

15. Mommersteeg MTM, Hoogaars WMH, Prall OWJ, et al. Molecular pathway for the localized formation of the sinoatrial node. Circ Res 2007;100:354-362.

16. $\mathrm{Yu} \mathrm{H}, \mathrm{Wu} \mathrm{J}$, Potapova I. A beta subunit for the $\mathrm{HCN}$ ion channel subunit family enhances expression and speeds activation. Circ Res 2001;88:E84-E87.

17. Schweizer P, Yampolsky $\mathrm{P}$, Malik R. Transcription profiling of $\mathrm{HCN}$ channel isotypes throughout mouse cardiac development. Basic Res Cardiol 2009;104:621-629.

18. Clubb Jr FJ, Bishop SP. Formation of binucleated myocardial cells in the neonatal rat. An index for growth hypertrophy. Lab Invest 1984;50:571-577.

19. Schmid G, Pfitzer P. Mitoses and binucleated cells in perinatal human hearts. Virchows Arch B Cell Pathol Incl Mol Pathol 1985;48:59-67.

20. Clubb J, Penney DG, Baylerian MS, et al. Cardiomegaly due to myocyte hyperplasia in perinatal rats exposed to $200 \mathrm{ppm}$ carbon monoxide. J Mol Cell Cardiol 1986;18:477-486.

21. Zardoya R, Arouheif E, Meyer A. Evolution and orthology of hedgehog genes. Trends Genet 1996;12:496-497.

22. Chiang $C$, Litingtung $Y$, Lee $E$, et al. Cyclopia and defective axial patterning in mice lacking Sonic hedgehog gene function. Nature 1996;383:407-413.

23. Tsukui T, Capdevila J, Tamura K, et al. Multiple left-right asymmetry defects in Shh(-/-) mutant mice unveil a convergence of the Shh and retinoic acid pathways in the control of Lefty-1. Proc Natl Acad Sci USA 1999;96:11376-11381.

24. Motoyama J, Liu J, Mo R, et al. Essential function of Gli2 and Gli3 in the formation of lung, trachea and oesophagus. Nat Genet 1998; 20:54-57.

25. Goodrich LV, IMG SRC, Higgins KM, et al. Altered neural cell fates and medulloblastoma in mouse patched mutants. Science 1997;277: 1109-1113.

26. Gianakopoulos PJ, Skerjanc IS. Hedgehog signaling induces cardiomyogenesis in P19 cells. J Biol Chem 2005;280:21022-21028.

27. Pende $M, U m$ SH, Mieulet $V$, et al. S6K1-/-/S6K2-/- mice exhibit perinatal lethality and rapamycin-sensitive $5^{\prime}$-terminal oligopyrimidine mRNA translation and reveal a mitogen-activated protein kinase-dependent S6 kinase pathway. Mol Cell Biol 2004;24:3112-3124.

28. Nau PN, Van Natta T, Ralphe JC, et al. Metabolic adaptation of the fetal and postnatal ovine heart: regulatory role of hypoxia-inducible factors and nuclear respiratory factor-1. Pediatr Res 2002;52: 269-278.

29. Iheagwara KN, Thom SR, Deutschman CS, et al. Myocardial cytochrome oxidase activity is decreased following carbon monoxide exposure. Biochim Biophys Acta (BBA) 2007;1772:1112-1116.

30. Guo W, Kamiya K, Hojo M, et al. Regulation of Kv4.2 and Kv1.4 K ${ }^{+}$ channel expression by myocardial hypertrophic factors in cultured newborn rat ventricular cells. J Mol Cell Cardiol 1998;30: 1449-1455.

31. Guo W, Kamiya K, Cheng J, et al. Changes in action potentials and ion currents in long-term cultured neonatal rat ventricular cells. Am J Physiol 1996;271:C93-102.

32. Subramaniam A, Jones WK, Gulick J, et al. Tissue-specific regulation of the alpha-myosin heavy chain gene promoter in transgenic mice. J Biol Chem 1991;266:24613-24620.

33. Reiser PJ, Westfall MV, Schiaffino $S$, et al. Tension production and thinfilament protein isoforms in developing rat myocardium. Am J Physiol 1994;267:H1589-H1596.

34. Lynch JM, Chilibeck K, Qui Y, et al. Assembling pieces of the cardiac puzzle; calreticulin and calcium-dependent pathways in cardiac 
development, health, and disease. Trends Cardiovasc Med 2006; 16:65-69.

35. Nakamura K, Robertson M, Liu G, et al. Complete heart block and sudden death in mice overexpressing calreticulin. J Clin Invest 2001;107:1245-1253.

36. Reed TD, Babu GJ, Ji Y, et al. The expression of SR calcium transport ATPase and the $\mathrm{Na}^{+} / \mathrm{Ca}^{2+}$ exchanger are antithetically regulated during mouse cardiac development and in hypo/hyperthyroidism. J Mol Cell Cardiol 2000;32:453-464.

37. Wiese C, Grieskamp T, Airik R, et al. Formation of the sinus node head and differentiation of sinus node myocardium are independently regulated by Tbx18 and Tbx3. Circ Res 2009;104:388-397.

38. Di Giovanni V, Cagiano R, De Salvia MA, et al. Neurobehavioral changes produced in rats by prenatal exposure to carbon monoxide. Brain Res 1993;616:126-131.

39. Jeron A, Mitchell GF, Zhou J, et al. Inducible polymorphic ventricular tachyarrhythmias in a transgenic mouse model with a long Q-T phenotype. Am J Physiol Heart Circ Physiol 2000;278: $\mathrm{H} 1891-\mathrm{H} 1898$.

40. Liu N, Colombi B, Memmi $M$, et al. Arrhythmogenesis in catecholaminergic polymorphic ventricular tachycardia: insights from a RyR2 R4496C knock-in mouse model. Circ Res 2006;99:292-298.
41. Stillitano F, Sartiani $L$, DePaoli $P$, et al. Expression of the hyperpolarization-activated current, $\mathrm{I}(\mathrm{f})$, in cultured adult rat ventricular cardiomyocytes and its modulation by hypertrophic factors. Pharmacol Res 2008;57:100-109.

42. Luo $\mathrm{X}$, Lin $\mathrm{H}$, Pan $\mathrm{Z}$, et al. Down-regulation of miR-1/miR-133 contributes to re-expression of pacemaker channel genes HCN2 and HCN4 in hypertrophic heart. J Biol Chem 2008;283:20045-20052.

43. Barbuti A, Terragni B, Brioschi $C$, et al. Localization of $f$-channels to caveolae mediates specific beta2-adrenergic receptor modulation of rate in sinoatrial myocytes. J Mol Cell Cardiol 2007:42:71-78.

44. Bosman AG, Sartiani L, Spinelli V, et al. Expression and subcellular localization of f-channels in early human development using hESCderived cardiomyocytes. Eur Heart J 2009;30(Abst Suppl):501.

45. Omalu $\mathrm{Bl}$, Lindner $\mathrm{JL}$, Janssen $\mathrm{JK}$, et al. The role of environmental factors in the causation of sudden death in infants: two cases of sudden unexpected death in two unrelated infants who were cared for by the same babysitter. J Forensic Sci 2007;52:1355-1358.

46. Di Cera E, Doyle ML, Morgan MS, et al. Carbon monoxide and oxygen binding to human hemoglobin F0. Biochemistry 1989;28:2631-2638.

47. Malik S, Cleves MA, Honein MA, , et al., and the National Birth Defects Prevention Study. Maternal smoking and congenital heart defects. Pediatrics 2008;121:e810-e816. 